\title{
Non-verbal IQ and change in restricted and repetitive behaviors throughout childhood in autism: A longitudinal study using the Autism Diagnostic Interview-Revised.
}

Valérie Courchesne ( $\nabla$ valerie.courchesne@gmail.com )

McGill University https://orcid.org/0000-0001-7768-5448

Rachael Bedford

King's College London

Andrew Pickles

King's College London

Eric Duku

McMaster University

Connor Kerns

UBC: The University of British Columbia

Pat Mirenda

UBC: The University of British Columbia

Terry Bennett

McMaster University

Stelios Georgiades

McMaster University

Isabel Smith

Dalhousie University

Wendy Ungar

University of Toronto

Tracy Vaillancourt

University of Ottawa

Anat Zaidman-Zait

Tel-Aviv University

Lonnie Zwaigenbaum

University of Alberta

Peter Szatmari

University of Toronto

Mayada Elsabbagh

McGill University 


\section{Pathways Team}

McGill University

\section{Research}

Keywords: Autism, longitudinal, restricted, repetitive, behaviors, interest, intelligence, ADI-R, Wechsler

Posted Date: December 16th, 2020

DOI: https://doi.org/10.21203/rs.3.rs-126244/v1

License: (c) (1) This work is licensed under a Creative Commons Attribution 4.0 International License. Read Full License

Version of Record: A version of this preprint was published at Molecular Autism on August 14th, 2021. See the published version at https://doi.org/10.1186/s13229-021-00461-7. 


\section{Abstract}

Background: Restricted and repetitive behaviors are one of the characteristic features of Autism Spectrum Disorder. This domain of symptoms includes a broad range of behaviors. There is a need to study each behavior individually to better understand the role they each have in the development of autistic children. Moreover, there are currently no longitudinal studies investigating change in these behaviors over development.

Methods: The goal of the present study was to explore the association between age and non-verbal IQ (NVIQ) on 15 RRB symptoms included in the Autism Diagnostic Interview-Revised (ADI-R) over time. A total of 205 children with ASD were assessed using the ADI-R at time of diagnosis, at age 6 years, and at age 11 years, and with the addition of the Wechsler Intelligence Scales for Children - Fourth Edition (WISC-IV) at age 8 years.

Results: The proportion of children showing each RRB tended to diminish with increasing age $(p<0.001)$, except for sensitivity to noise and circumscribed interests, where the proportion increased over time. Although there was no significant main effect of NVIQ $(p=0.101)$, there was a significant interaction between age and NVIQ $(p<0.01)$. This was mainly driven by Difficulties with change in routine, for which higher NVIQ was associated with the prevalence of the behavior remaining relatively stable with age, while lower NVIQ was associated with the behavior becoming more prevalent with age.

Limitations: The study focused on the presence/absence of each RRB but did not account for potential changes in frequency or severity of the behaviors over development. Furthermore, some limitations are inherent to the measures used. The ADI-R relies on parent-report and hence has some level of subjectivity, while the Wechsler intelligence scales can underestimate the intellectual abilities of some autistic children.

Conclusions: These results confirm that specific RRBs are differentially linked to age and NVIQ. Studying RRBs individually is a promising approach to better understand how RRBs change over the development of autistic children and are linked to other developmental domains.

\section{Background}

Restricted and repetitive behaviors (RRBs) are central to the phenotype and diagnosis of Autism Spectrum Disorder (ASD) (DSM-5 1), RRBs are considered «positive» symptoms of autism as they include behaviors that the person does more often or differently, instead of an absence of behaviors, like those described in the social-communication domain (2). These symptoms include stereotyped and repetitive motor movements (e.g., finger mannerisms, arm movements or hand flapping), stereotyped speech (e.g., echolalia), insistence on sameness (e.g., rigid adherence to certain routines or rituals), intense interests in specific areas such as dinosaurs or public transport maps, and special skills (e.g., calendar calculation or memory for routes) $(1,3,4)$. RRBs are observed in autistic children of all intelligence level but not at every age and not in the same proportion (2). 
Despite their higher prevalence in autism, RRBs are also observed in populations diagnosed with other conditions such as intellectual disability, Tourette's syndrome, obsessive-compulsive disorder (5-7) and in typically developing children (i.e. 5, 6, 8). These behaviors are perceived differently in developmental conditions and in typical development. For example, early on in infancy, stereotyped motor behaviors are thought to be intrinsic to the development of complex functional motor behaviors in typically developing children $(9,10)$. These stereotypies also serve a communication function since they are more frequently observed during child-caregiver interactions and diminish as verbal abilities augment in typically developing children (10-12). Furthermore, for typically developing children, these repetitive behaviors are a way to adapt to one's environment and by repeating, classifying, and ordering, the brain is able to organize information from the environment (13). Attempts to understand the role of these behaviors in typically developing children could be informative for clarifying the role of RRBs in autism or in other conditions. Indeed, the role of stereotypies in the development of motor skills or the possibility that at least some repetitive behaviors serve adaptive purposes in typical development are promising avenues to explore when trying to understand the role some of these behaviors might have in the development of individuals with autism.

In order to understand the role of RRBs in autism, research to-date has taken three approaches. One approach is to study the domain of RRBs symptoms as a whole, combining all individual behaviors and investigating whether presenting more or less symptoms in that domain is positively or negatively related to other domains of functioning, such as intelligence, language abilities, or adaptive functioning; and how the whole domain changes with age. The second approach is to group individual RRBs into categories, usually derived from factor analysis, and the third is to study each RRB individually.

When studied as a whole, RRBs increase throughout preschool years but then diminish later in childhood $(14,15)$. This is especially the case for autistic individuals with higher cognitive and language abilities (16). Moreover, the presence of more RRBs is generally linked to lower adaptive functioning (17), language abilities (18) and intelligence (19).

A different pattern of results emerges when RRBs are investigated using factor analyses. The number of factors usually identified depends on the specific RRB measure and the age range of the sample. For example, using the Autism Diagnostic Interview-Revised (ADI-R, 20), when participants older than 3 years of age are included, a third factor, circumscribed interests (CI), emerges in addition to the Repetitive and Sensory Motor (RSM) and Insistence on Sameness (IS) factors (e.g. 21). Using the Repetitive Behavior Scale - Revised (RBS-R, 22), some researchers have identified a three-factor model (23), while others have found five- $(21,23)$ or six-factor models $(24)$.

The links between the categories of RRBs derived from factor analysis and other developmental domains are somewhat inconsistent (see: 16 for a review). For example, the score on the RSM factor derived from the ADI-R was shown to be negatively correlated to adaptive functioning (3), while none of the factors derived from the RBS-R were shown to correlate to the developmental level (23). The IS factor is often not correlated to IQ $(21,25)$, but the corresponding factor derived from the RBS-R is negatively correlated to 
NVIQ (17). Taken together, studying RRBs as a whole, or even as factors has not revealed a consistent pattern of results across studies. A more fine-grained exploration of the RRB domain is warranted in order to clarify the nature of the links between developmental domains and the different behaviors included in the RRB domain. To-date, few studies have looked at individual RRBs. Bishop, Richler (2) explored the association between each of the 15 RRB items from the ADI-R, age, and NVIQ in a large cross-sectional study of 830 autistic children ranging in age from 3 to 11 years. Although the association between NVIQ and the prevalence of specific RRBs was generally negative (i.e. the behavior was more prevalent in groups of children with lower NVIQ), two specific RRBs were more prevalent in children with higher NVIQ, namely Compulsions/rituals and Circumscribed interests. These positive and negative associations with NVIQ tended to become stronger with age. As for the severity of each behavior, it was either not associated with NVIQ or was related in the same fashion as was prevalence (2).

This approach illustrates that taking a more global or factor analytic approach to RRBs could lead to specific behaviors being combined despite their differential associations with intelligence, adaptive behaviors, language level, or age $(2,19)$. These findings also suggest that the presence of some individual behaviors may be more strongly linked to developmental level and intelligence, while others might be more specific to the autism phenotype $(2,26)$. The presence of a specific RRB early in development might not have the same implications as the presence of this same behavior later on in development. Indeed, the presence of a behavior at a young age may not necessarily be linked to NVIQ at that particular time in development. However, if that same behavior is still present as the child gets older, it can become an indicator of a lower IQ or level of functioning (2).

The above findings suggest that RRBs include several distinct features that likely have different underlying etiologies and manifest differently over development, thus warranting investigation of individual behaviors. However, to date, cross sectional studies have provided only snapshots of development across rather than within individuals over time. The current study, builds on the previous study from Bishop, Richler (2), by longitudinally examining specific RRBs included in the ADI-R in relation to age and NVIQ in an inception cohort of children followed from age of diagnosis to around 11 years of age.

\section{Methods}

\section{Participants}

Participants for this study were drawn from the Pathways in ASD study, an inception cohort of 421 families recruited across five provinces in Canada aimed at better understanding developmental trajectories in autism. The study was approved by each of the local Research Ethics Boards and informed consent was obtained from all participating families. Children in the cohort met ASD criteria on the Autism Diagnostic Observation Schedule (ADOS; 27), on the Social and at least one other domain on the ADI-R (20), as well as DSM-IV criteria (APA, 28) according to expert clinical judgement. Exclusion criteria 
included any known genetic abnormality, neuromotor disorder (such as cerebral palsy), or severe hearing or vision disorders. Only one child per family with ASD was included.

The ADI-R was administered at three time points (time of diagnosis $=T 1$; age 6 years $=T 4$; age 11 years $=$ T8). In the current study, we included all participants with at least the first ADI-R time point and a valid NVIQ $(n=205)$. The Perceptual Reasoning Index (PRI) of the Wechsler Intelligence Scales for Children (WISC-IV; Wechsler, 2003) was used as a proxy of NVIQ (see WISC-IV section below and Table 1 for more details and Table $\mathrm{S} 1$ for site distribution).

Table 1

Number of participants, age and scores for each measure and time point.

\begin{tabular}{|lllll|}
\hline Measure & T1 & T4 & T6 & T8 \\
\hline $\mathrm{N}$ & 205 & 199 & 205 & 157 \\
\hline Age & $3.43(.75)$ & $6.61(.33)$ & $8.73(.21)$ & $10.73(.21)$ \\
\hline ADI-R RRB & $4.52(2.30)$ & $4.07(2.57)$ & - & $3.66(2.35)$ \\
\hline WISC-IV PRI & - & - & $91.28(19.50)$ & - \\
\hline $\begin{array}{l}\text { Note. Numbers in parenthesis are standard deviations. ADI-R = Autism Diagnostic Interview -Revised. } \\
\text { Numbers reported are mean total scores for the RRB domain using current scores. WISC-IV PRI = } \\
\text { Wechsler Intelligence Scales for Children- Fourth Edition, Perceptual Reasoning Index. Number } \\
\text { reported is the mean Standard Score. }\end{array}$ & \\
\hline
\end{tabular}

Measures

Autism Diagnostic Interview-Revised (ADI-R)

The ADI-R $(20,29)$ is a comprehensive caregiver interview, administered by a trained clinician. It is one of the gold standard instruments used to discriminate between children with and without autism (30) and to differentiate between ASD and an intellectual disability or a language impairment (20). It covers the different domains of autistic symptoms, one of which is the Restricted and Repetitive Behaviors domain. In total, 15 ADI-R items relevant for RRBs were included in the current analysis (see table S2 for a list). During the ADI-R interview, parents indicated whether the behavior was present in the past, «ever», and if it was present at time of administration, «current». Given the longitudinal design of the study, only the «current» scores of the RRBs items were included in the analysis.

Scores on the ADI-R items vary between 0 and 3 , where 0 indicates the absence of the behavior or no abnormality, 1 indicates the behavior is present or abnormal to some degree, 2 indicates the behavior is present and frequent/severe/abnormal enough to impair functioning to some degree and 3 indicates the behavior is present and is sufficiently intrusive or abnormal to significantly impair functioning. In line with what was done by Bishop, Richler (2) in order to examine prevalence separately from severity, a presence/absence score was computed by recoding scores of 2 and 3 into « 1 ». All included ADI-R items were therefore transformed into binary variables where « 0 » indicates the behavior is absent and « 1 » 
indicates it is present. As the current study is a first attempt to study individual RRBs using a longitudinal design and because the examination of severity in relation to NVIQ and age in Bishop's 2006 study did not yield any additional information, the analysis considers only the presence/absence of a behavior.

The ADI-R scoring system typically recodes scores of ' 8 ' as ' 0 ', indicating that no atypicality was observed. However, this conflates children where RRBs could have been observed but were not, with those who did not yet have the linguistic ability to show RRBs. Coding these children's scores of ' 8 ' as missing is therefore the more conservative approach in that it does not assign the presence or absence of RRBs to these children. Therefore, items with scores of ' 8 ' were recoded in the following manner. For Stereotyped Speech and Verbal Rituals children who were coded '8' (i.e. they did not have enough phrase speech to make assessment possible), were treated as missing in this analysis. Similarly, for Circumscribed Interests, children below the age of three who were coded as ' 8 ' were recoded as missing. In addition, for Unusual Attachment to Objects, children who were coded as ' 6 ' or ' 7 ' (i.e. "attachment to usual objects after 5-years-old or to such intensity that it interferes with social functioning" or "series of short lasting attachment to unusual objects") were recoded into ' 1 ' as these indicate a certain level of atypicality in the behavior, despite not being considered in the ADI-R algorithm (29).

Wechsler Intelligence Scale for Children - Fourth Edition (WISC-IV)

The standard score obtained on the Perceptual Reasoning Index (PRI) of the WISC-IV was used as a proxy of NVIQ. This test was administered at T6 (8-years-old) and was determined the best available proxy of NVIQ for this cohort. Tests administered at previous time points are more prone to be influenced by testability issues (i.e. difficulties following directions, understanding spoken language, etc.) and therefore might not be as representative of non-verbal reasoning abilities (31). Including the latest time-point for which IQ was available was also motivated by the fact that IQ is more stable in later childhood (Flanagan et al., 2015).

\section{Statistical Analysis}

Data were analysed with binary logistic Generalised Estimating Equations (GEE) in Stata v15 (32). GEE was chosen as it 1) accounts for missingness (i.e. GEE uses all available data from each child, and accounts for selective attrition associated with included covariates and factors); 2 ) is robust to the misspecification of the pattern of correlation among ADI items through the use of robust standard errors for the calculation of the Wald statistics; and 3) allows flexible inclusion of age as a time varying covariate, which represents both age at diagnosis between subjects, as well as within subject change in RRBs over time. Models had a logit link function and an exchangeable correlation matrix with robust standard errors; binary ADI score was the outcome. The first model included main effects of ADI item as a multivariate measure $(83,72,73,71,69,77,78,33,39,74,75,70,67,76,68)$, IQ at T6, Age (of the child at each time point, included as a time varying covariate) and controlled for Site (Montreal, Halifax, Hamilton, Vancouver, Edmonton) and age at T1 (beginning of the study). The following two and three-way interactions were then added as a secondary step: $A D I$ itemAge, $A D I$ itemIQ, ADI itemAge*IQ. To break 
down the main effect of ADI item, separate GEE models were then run for each ADI item, including main effects of IQ, Age and Site; an Age*IQ interaction was then added as a second step. The testparm command in Stata was used to assess the overall significance of main effects in the model. For the analysis split by ADI item, two types of results are reported. First, given that we had no prior hypothesis regarding any specific ADI items' link with age and/or NVIQ, Bonferroni corrections were deemed appropriate (adjusted level of significance, $0.05 / 15=0.0033$ ) However, this is a highly conservative approach that could lead to false negatives, thus we also reported Benjamini-Hochberg corrected results using a False Discovery Rate (FDR) of 0.05 . For a complete list including non-corrected results for all items see table $\mathrm{S} 2$ in supplementary material.

\section{Results}

\section{Effect of age}

Results from the main GEE model, showed a significant main effect of age (Wald $\chi^{2}(1)=28.40, p<0.001$ ) where the overall presence of ADI-R RRB decreased over time across the whole sample. There was also a significant interaction between age and ADI-R item (Wald $\chi^{2}(14)=160.74, p<0.001$, See Table 2). Separate GEE models for each ADI-R item using a Bonferroni correction for multiple comparisons, showed that this interaction was driven by certain RRBs significantly decreasing with age (Unusual Sensory Interest, Repetitive Use of Objects, Other Complex Mannerisms and Unusual Preoccupations) while others significantly increased with age (Circumscribed Interests, Sensitivity to Noise). BenjaminiHochberg corrections led to the identification of one additional item that significantly decreased with age (Unusual Attachment to Objects) (see Fig. 1).

Figure 1. Prevalence of each ADI-R item at each time point. ${ }^{* *}=$ item with a significant effect of age after Bonferroni corrections. * item with a significant effect of age after Benjamini-Hochberg corrections. T1 = time of Diagnosis, $\mathrm{T} 4=6$ years old, $\mathrm{T} 8=11$ years old.

\section{Effect of NVIQ}

There was no main effect of NVIQ (Wald $\left.\chi^{2}(1)=2.70, p=0.101\right)$ in the main GEE model. However, there was a significant 2-way interaction between NVIQ and ADI-R item (Wald $\left.\chi^{2}(14)=30.22, p=0.007\right)$. There was also a 3-way interaction between NVIQ, age, and ADI-R items (Wald $\chi^{2}(15)=32.60, p=0.005$ ). See Table 2 for the detail of all the model results and results for covariates. Separate GEE models split by ADI$\mathrm{R}$ item and level of significance corrected for multiple comparisons using Bonferroni, showed a significant interaction between age and NVIQ only for Difficulties with Change in Routine, which increased over time in children with lower NVIQ. Benjamini-Hochberg corrected results include three additional items showing a significant interaction between age and NVIQ (Unusual Preoccupations, Resistance to Change and Compulsions and Rituals). Despite the fact that NVIQ was included in the model as a continuous variable, to allow for a graphic representation, IQ groups were split according to the median (Median standard score of 93, Range 51-134) and are presented in Fig. 2. 
Figure 2. Percentage of children at each time point for each item presenting a significant interaction between age and NVIQ. The item on the left was significant following Bonferroni correction and the three additional items on the right were significant only when using Benjamini-Hochberg corrections. Lower $\mathrm{NVIQ}=<93 ; n=101$, Higher NVIQ = >93; $n=104 . \mathrm{T} 1=$ time of Diagnosis, $\mathrm{T} 4=6$ years old, $\mathrm{T} 8=11$ years old.

Table 2

GEE model results.

\begin{tabular}{|ll|}
\hline ADI-R item & Wald $\chi^{2}(14)=624.32, p<0.001$ \\
\hline Site & Wald $\chi^{2}(4)=25.87, p<0.001$ \\
\hline Age & Wald $\chi^{2}(1)=28.40, p<0.001$ \\
\hline NVIQ & Wald $\chi^{2}(1)=2.70, p=0.101$ \\
ADI-R item * Age & Wald $\chi^{2}(14)=160.74, p<0.001$ \\
ADI-R item * NVIQ & Wald $\chi^{2}(14)=30.22, p=0.007$ \\
ADI-R item * Age * NVIQ & Wald $\chi^{2}(15)=32.60, p=0.005$ \\
\hline
\end{tabular}

\section{Discussion}

We conducted a novel longitudinal investigation of individual Restricted and Repetitive Behaviors in a cohort of autistic children followed from age of diagnosis to 11 years of age. The association between the presence or absence of each of the 15 RRBs items from the ADI-R and age as well as NVIQ was explored. Overall, we found a decreasing prevalence for most RRBs throughout childhood, with the exception of Sensitivity to Noise and Circumscribed Interest, the prevalence of which significantly increased with age. We found no main effect of NVIQ on the prevalence of any of the ADI-R items. There was however a significant interaction between Difficulties with Change in Routine, age and NVIQ, indicating that the change in the prevalence of these behaviors with age varied depending on children's NVIQs. Less conservative corrections led to 3 additional items showing a significant interaction with age and NVIQ, namely Unusual Preoccupations, Resistance to Change and Compulsion and rituals. These results illustrate that when examining RRBs, item level analyses may be helpful before grouping behaviors into higher level domains, as their associations with age and intelligence are complex and heterogeneous.

The association that we found between age and the prevalence of RRBs parallels the decrease observed from preschool age to school age when considering all behaviors included in the RRB domain as a single construct $(15,33,34)$ or when studying the change with age on the RSM factor derived from the ADI-R (4, 21). However, the item by item analysis used in the current study demonstrates that this decrease is not present across all RRBs. Indeed, we found a significant decrease for only 4 behaviors of the 15 RRB items 
analysed. The prevalence of 9 behaviors was not significantly associated with age and for the other 2 behaviors, the prevalence significantly increased with age. The use of less conservative correction led to only one additional item significantly decreasing with age (Unusual attachment to objects). Given that our measure of prevalence was based on absence or presence of the behavior, the significant effect of age indicates that for those items, there was a significant proportion of children who went from presenting the behavior (scores of 1-2 or 3 ) to not presenting it at all (score of 0 ) or the opposite. Such changes are not only statistically significant, but also of high clinical significance as it means going from presenting an atypical behavior to presenting no atypicality at all or going from a completely normal behavior to an atypical one. Taken together, the results of the current study suggest that, to understand the developmental pathways of RRBs in autism, an item based approach is warranted, at least as a first step.

The differential effect of age on the prevalence of RRBs during childhood could be the result of some behaviors morphing into other behaviors. For example, as the child gets older, Repetitive Use of Objects could transform to become a Circumscribed Interest. This hypothesis is consistent with our results as well as with Bishop's previous findings that Repetitive use of objects tends to diminish with age while Circumscribed Interests tends to augment (2). To directly test this hypothesis that RRBs evolve through development, studies using latent transition models are needed. It is also possible that some RRBs represent a step of the autistic development and are therefore transient by nature. For example, a similar hypothesis was put forward regarding language development in autism. It was proposed that Verbal Rituals and Stereotyped Speech such as echolalia are milestones of language development and thus are more frequent when the child first starts speaking and then diminish and disappear as the child develops more oral language $(35,36)$. A similar hypothesis could explain the fact that we found an increase in the prevalence of Sensitivity to Noise during childhood. It is possible that the development and improvement of spoken language allows many autistic children to better describe their experience to caregivers, who then are more prone to report this sensitivity during the ADI-R interview.

With respect to NVIQ, while we found no significant main effect on the prevalence of the ADI-R items, NVIQ did interact with age for one specific behavior; Difficulties with Change in Routine. This result is consistent with results from Bishop's cross-sectional study (2), which found differences between NVIQ groups in the prevalence of certain RRBs only in older children, with the exception of Compulsion and Rituals that differed between NVIQ groups only at earlier age (3-6 years). In the present study, Difficulties with Change in Routine followed a path characterized by the behavior being less prevalent in children with lower NVIQ at age of diagnosis and then becoming more prevalent in these children as they grew older, while in children with higher NVIQ, it remained stable during the same time period. The path for three additional items (Resistance to Change and Compulsion and rituals and Unusual preoccupations) was similar and significant when using Benjamini-Hochberg corrections, but was characterized by a decrease in prevalence with age in children with higher NVIQ (instead of stability) while the prevalence was stable or slightly increased in children with lower NVIQ . 
In typically developing children, it was suggested that rituals, compulsive-like behaviors and sameness behaviors are a way to adapt and to gain a sense of control over one's environment (37). It is therefore possible that they have a similar role in autistic development. Hence, autistic children with higher NVIQ may display these behaviors sooner than those with lower NVIQ and then a proportion of children with higher NVIQ learn other ways to adapt to the environment, while most children with lower NVIQ do not and are therefore more likely to continue presenting these behaviors.

Taken together, these results highlight the importance of considering age when investigating the association between RRBs and intelligence or other developmental indicators. RRBs can be observed in autistic children of all IQ levels, but not necessarily at every age. This interaction is important to consider when making a prognosis about the evolution of RRBs, for example. Our results also show that investigation of individual behaviors over time is essential in order to truly understand how each behavior evolves with age and how this evolution differs depending on other individual characteristics such as intelligence, language or adaptive levels.

Limitations. Our findings need to be considered against a number of inherent limitations related to measurement of RRBs in autism. Indeed, here we focused solely on the presence or absence of RRBs as measured through the ADI-R, which led to an investigation of the change in prevalence of each RRB, without accounting for either frequency or severity of each behavior. As a future step, it would be interesting to investigate whether the changes in severity or frequency parallel those we found in prevalence. However, the field of autism is lacking a good measure of RRB frequency that is independent of their functional impact on the individual. Only observational studies provide such measures of frequency/duration $(16,38)$. The results of this study also need to be interpreted while taking into consideration that the ADI-R is a parent reported measure. It is therefore possible that some behaviors were present, but not noted or noticed by parents.

Other limitations include the measurement of intelligence in autism. Intelligence measured through conventional IQ tests tends to underestimate the intellectual potential of most autistic children $(31,39)$. The choice to use PRI at age 8 as a measure of NVIQ in this study aimed at minimizing the effect of testability and reducing the risks of underestimation. It is however still possible that the NVIQ of certain of our participants remained underestimated, especially in the children who were less verbal and for whom intelligence testing is often more challenging $(40,41)$.

Finally, another limitation is that we did not investigate the association between age and RRBs in relation to other developmental indicators, such as language development or changes in adaptive abilities. This would be an interesting future step. In addition, future studies following the development of RRBs in children after 11 years old and into adolescence and adulthood could further inform those associations. Investigating stability and change in later development would allow to investigate the transient or permanent nature of specific RRBs in autism development. For example, it would help determine if the presence of certain RRBs in children with lower NVIQ follow the same pathway as in children with higher NVIQ, but with a delay or if it tends to persist in adolescence and adulthood in these children. 


\section{Conclusions}

This study confirms previous findings showing that most RRBs tend to diminish or even disappear from age of diagnosis to later childhood, especially in children with higher NVIQ. However, not all behaviors follow this developmental trajectory. Indeed, while some RRBs diminish over time, others increase in prevalence with age, and these changes vary in function of NVIQ. While some RRBs are likely an inherent part of autistic development, more research is needed to fully understand how each of them evolves over time, and how this evolution is associated with other aspects of development.

\section{Declarations}

Ethics approval and consent to participate: This study was approved by each of the local Research Ethics Boards and informed consent was obtained from all participating families.

\section{Consent for publication: NA}

Availability of data and materials: The dataset analysed during the current study are not publicly available due to the data analysed being composed of sensitive private information that could compromise the privacy of the research participants, but are available from the corresponding author on reasonable request.

Competing interests: The authors declare having no financial or non-financial competing interests.

Funding: Authors acknowledge funding by: The Azrieli Centre for Autism Research (ACAR), The Canadian Institutes of Health Research, Fonds de Recherche du Québec, Kids Brain Health Network (formerly NeuroDevNet), Autism Speaks (US), Government of British Columbia, Alberta Innovates Health Solutions, and the Sinneave Family Foundation. AP received additional support from the NIHR KCL/South London and Maudsley NHS Foundation Trust Biomedical Research Centre and Senior Investigator Award NF-SI0617-10120. RB is supported by a King's Prize Fellowship (204823/Z/16/Z).

Author's contribution: VC contributed to the data collection, analysis, interpretation manuscript writing, and revisions. RB conducted the statistical analysis and modelling and contributed to the interpretation of results and manuscript writing and revisions. AP contributed to the data analysis and interpretation. ED contributed to the study design and manuscript revisions. CK contributed to the data interpretation and manuscript revisions. PM, TB, SG, IS, WJU, TV, CW, AZZ, LZ and PZ, and ME contributed to the study design, data collection and manuscript revisions.

Acknowledgements: The authors would like to thank Samantha Wunderlich and Myriam Beauchamp for their thoughtful comments and revisions on the manuscript. We would also like to thank Julie Scorah for her help with the choice of study measures. Thanks also to the QUIC workgroup for their help throughout the writing of this paper; Rackeb Tesfaye, Nicola Wright, Virginia Carter Leno, Hannah Pickard and Alana McVey. The authors also acknowledge the past and current members of the Pathways in ASD Study 
Team, who made equal contributions to the study. Finally we are extremely grateful to all the families participating in the Pathways in ASD study for their continued engagement in this project.

Availability of data and materials: Participants did not consent to making their data publicly available and it would be a violation of our ethics to openly share this confidential dataset. However, if researchers are interested in collaborating or learning more about Pathways in ASD data they can contact the corresponding author.

\section{References}

1. American Psychiatric Association. Diagnostic and Statistical Manual of Mental Disorders Fifth ed. Arlington: American Psychiatric Association; 2013.

2. Bishop SL, Richler J, Lord C. Association between restricted and repetitive behaviors and nonverbal IQ in children with autism spectrum disorders. Child neuropsychology. 2006;12(4-5):247-67.

3. Cuccaro ML, Shao Y, Grubber J, Slifer M, Wolpert CM, Donnelly SL, et al. Factor analysis of restricted and repetitive behaviors in autism using the Autism Diagnostic Interview-R. Child psychiatry and human development. 2003;34(1):3-17.

4. Bishop SL, Hus V, Duncan A, Huerta M, Gotham K, Pickles A, et al. Subcategories of restricted and repetitive behaviors in children with autism spectrum disorders. J Autism Dev Disord. 2013;43(6):1287-97.

5. DeLoache JS, Simcock G, Macari S. Planes, trains, automobiles-and tea sets: Extremely intense interests in very young children. Dev Psychol. 2007;43(6):1579.

6. Evans DW, Gray FL. Compulsive-like Behavior in Individuals with Down Syndrome: Its Relation to Mental Age Level, Adaptive and Maladaptive Behavior. Child Dev. 2000;71(2):288-300.

7. Leekam SR, Tandos J, McConachie H, Meins E, Parkinson K, Wright C, et al. Repetitive behaviours in typically developing 2-year-olds. J Child Psychol Psychiatry. 2007;48(11):1131-8.

8. Uljarević M, Arnott B, Carrington SJ, Meins E, Fernyhough C, McConachie H, et al. Development of restricted and repetitive behaviors from 15 to 77 months: Stability of two distinct subtypes? Dev Psychol. 2017;53(10):1859.

9. Shafer RL, Newell KM, Lewis MH, Bodfish JW. A cohesive framework for motor stereotypy in typical and atypical development: the role of sensorimotor integration. Front Integr Nuerosci. 2017;11:19.

10. Thelen E. Rhythmical behavior in infancy: An ethological perspective. Dev Psychol. 1981;17(3):237.

11. Berry K, Russell K, Frost K. Restricted and repetitive behaviors in autism spectrum disorder: A review of associated features and presentation across clinical populations. Current Developmental Disorders Reports. 2018;5(2):108-15.

12. Larkin F, Meins E, Centifanti LC, Fernyhough C, Leekam SR. How does restricted and repetitive behavior relate to language and cognition in typical development? Dev Psychopathol. 2017;29(3):863-74. 
13. Langen M, Durston S, Kas MJ, van Engeland H, Staal WG. The neurobiology of repetitive behavior:... and men. Neuroscience Biobehavioral Reviews. 2011;35(3):356-65.

14. Moore V, Goodson S. How well does early diagnosis of autism stand the test of time? Follow-up study of children assessed for autism at age 2 and development of an early diagnostic service. Autism. 2003;7(1):47-63.

15. Esbensen AJ, Seltzer MM, Lam KS, Bodfish JW. Age-related differences in restricted repetitive behaviors in autism spectrum disorders. J Autism Dev Disord. 2009;39(1):57-66.

16. Leekam SR, Prior MR, Uljarevic M. Restricted and repetitive behaviors in autism spectrum disorders: a review of research in the last decade. Psychological bulletin. 2011;137(4):562.

17. Gabriels RL, Cuccaro ML, Hill DE, Ivers BJ, Goldson E. Repetitive behaviors in autism: Relationships with associated clinical features. Res Dev Disabil. 2005;26(2):169-81.

18. Barrett S, Prior M, Manjiviona J. Children on the borderlands of autism: Differential characteristics in social, imaginative, communicative and repetitive behaviour domains. Autism. 2004;8(1):61-87.

19. Troyb E, Knoch K, Herlihy L, Stevens MC, Chen C-M, Barton M, et al. Restricted and Repetitive Behaviors as Predictors of Outcome in Autism Spectrum Disorders. J Autism Dev Disord. 2016;46(4):1282-96.

20. Lord C, Rutter M, Le Couteur A. Autism Diagnostic Interview-Revised: a revised version of a diagnostic interview for caregivers of individuals with possible pervasive developmental disorders. J Autism Dev Disord. 1994;24(5):659-85.

21. Lam KS, Bodfish JW, Piven J. Evidence for three subtypes of repetitive behavior in autism that differ in familiality and association with other symptoms. J Child Psychol Psychiatry. 2008;49(11):1193200.

22. Bodfish JW, Symons FJ, Parker DE, Lewis MH. Varieties of repetitive behavior in autism: Comparisons to mental retardation. J Autism Dev Disord. 2000;30(3):237-43.

23. Mirenda P, Smith IM, Vaillancourt T, Georgiades S, Duku E, Szatmari P, et al. Validating the repetitive behavior scale-revised in young children with autism spectrum disorder. J Autism Dev Disord. 2010;40(12):1521-30.

24. Martínez-González AE, Piqueras J. Validation of the Repetitive Behavior Scale-Revised in SpanishSpeakers Participants with Autism Spectrum Disorder. J Autism Dev Disord. 2018;48(1):198-208.

25. Richler J, Huerta M, Bishop SL, Lord C. Developmental trajectories of restricted and repetitive behaviors and interests in children with autism spectrum disorders. Dev Psychopathol. 2010;22(1):55-69.

26. Mottron L, Mineau S, Martel G, Bernier CS-C, Berthiaume C, Dawson M, et al. Lateral glances toward moving stimuli among young children with autism: Early regulation of locally oriented perception? Dev Psychopathol. 2007;19(01):23-36.

27. Lord C, Risi S, Lambrecht L, Cook EH Jr, Leventhal BL, DiLavore PC, et al. The Autism Diagnostic Observation Schedule-Generic: A standard measure of social and communication deficits associated with the spectrum of autism. J Autism Dev Disord. 2000;30(3):205-23. 
28. American Psychiatric Association. Diagnostic and Statistical Manual of Mental Disorders - Text Revision. Fourth ed. Washington, DC: American Psychiatric Association; 2000.

29. Le Couteur A, Lord C, Rutter M. The autism diagnostic interview-revised (ADI-R). Los Angeles, CA: Western Psychological Services. 2003:659 - 85.

30. Falkmer T, Anderson K, Falkmer M, Horlin C. Diagnostic procedures in autism spectrum disorders: a systematic literature review. Eur Child Adolesc Psychiatry. 2013;22(6):329-40.

31. Courchesne V, Girard D, Jacques $C$, Soulières I. Assessing intelligence at autism diagnosis: mission impossible? Testability and cognitive profile of autistic preschoolers. J Autism Dev Disord. 2019;49(3):845-56.

32. StataCorp. Stata Statistical Software: Release 15. In: Station C, editor. TX: Stata Corp LLC; 2017.

33. Charman T, Taylor E, Drew A, Cockerill H, Brown JA, Baird G. Outcome at 7 years of children diagnosed with autism at age 2: Predictive validity of assessments conducted at 2 and 3 years of age and pattern of symptom change over time. J Child Psychol Psychiatry. 2005;46(5):500-13.

34. Schertz HH, Odom SL, Baggett KM, Sideris JH. Parent-reported repetitive behavior in toddlers on the autism spectrum. J Autism Dev Disord. 2016;46(10):3308-16.

35. Roberts JM. Echolalia and language development in children with autism. Communication in autism. 2014;11:55-74.

36. Prizant BM, Fields-Meyer T. Uniquely human: A different way of seeing autism: Simon and Schuster; 2015.

37. Tregay J, Gilmour J, Charman T. Childhood rituals and executive functions. Br J Dev Psychol. 2009;27(2):283-96.

38. Harrop C, McConachie H, Emsley R, Leadbitter K, Green J. Restricted and Repetitive Behaviors in Autism Spectrum Disorders and Typical Development: Cross-Sectional and Longitudinal Comparisons. Journal of Autism \& Developmental Disorders. 2014;44(5).

39. Nader A-M, Courchesne V, Dawson M, Soulières I. Does WISC-IV Underestimate the Intelligence of Autistic Children? Journal of Autism and Developmental Disorders. 2014:1-8.

40. Akshoomoff N. Use of the Mullen Scales of Early Learning for the Assessment of Young Children with Autism Spectrum Disorders. Child Neuropsychol. 2006;12(4-5):269-77.

41. Courchesne V, Meilleur A-AS, Poulin-Lord M-P, Dawson M, Soulières I. Autistic children at risk of being underestimated: school-based pilot study of a strength-informed assessment. Molecular autism. 2015;6(1):12.

\section{Figures}




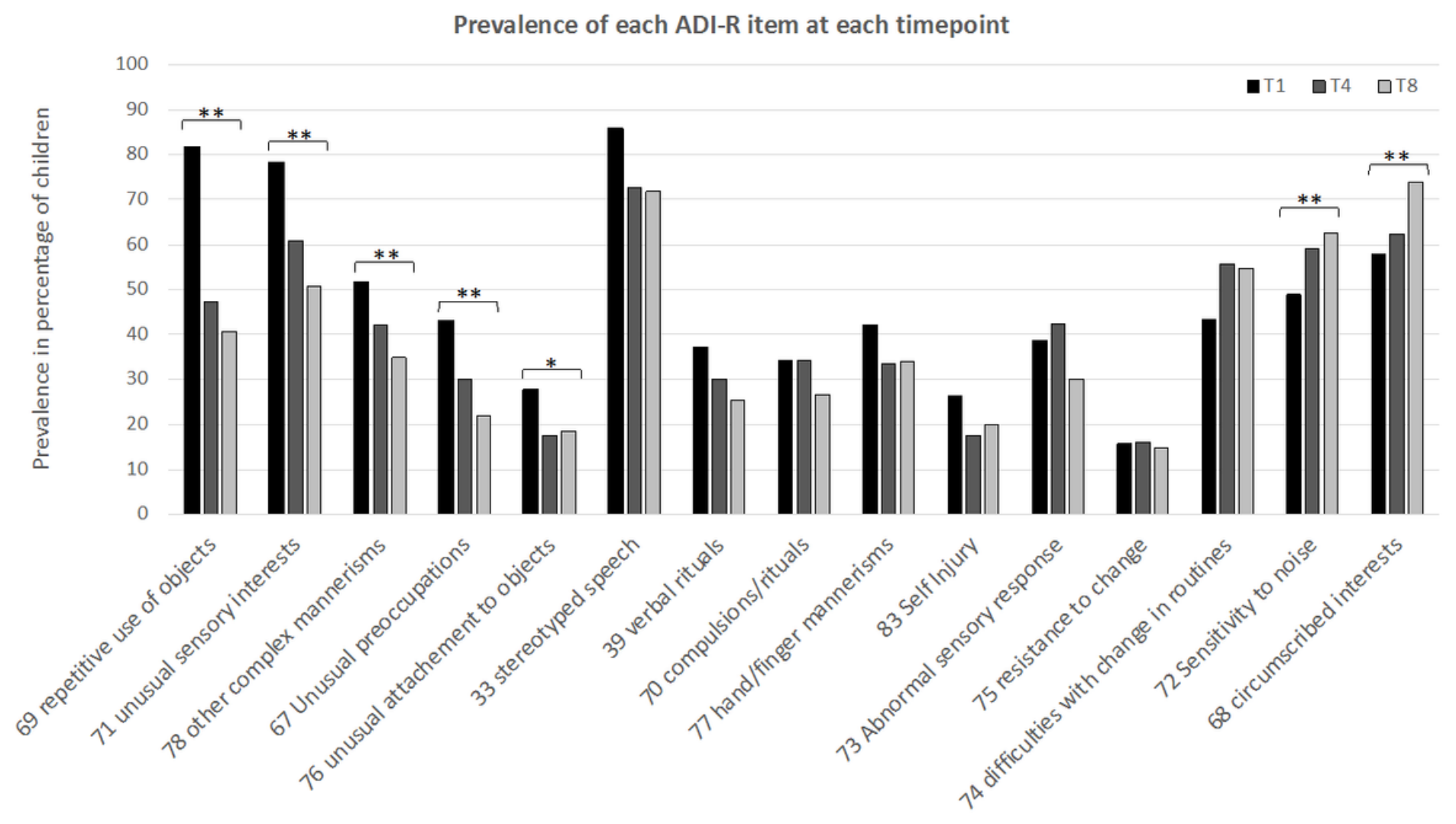

Figure 1

Prevalence of each ADI-R item at each time point. **=item with a significant effect of age after Bonferroni corrections. * item with a significant effect of age after Benjamini-Hochberg corrections. $T 1=$ time of Diagnosis, $T 4=6$ years old, $T 8=11$ years old. 


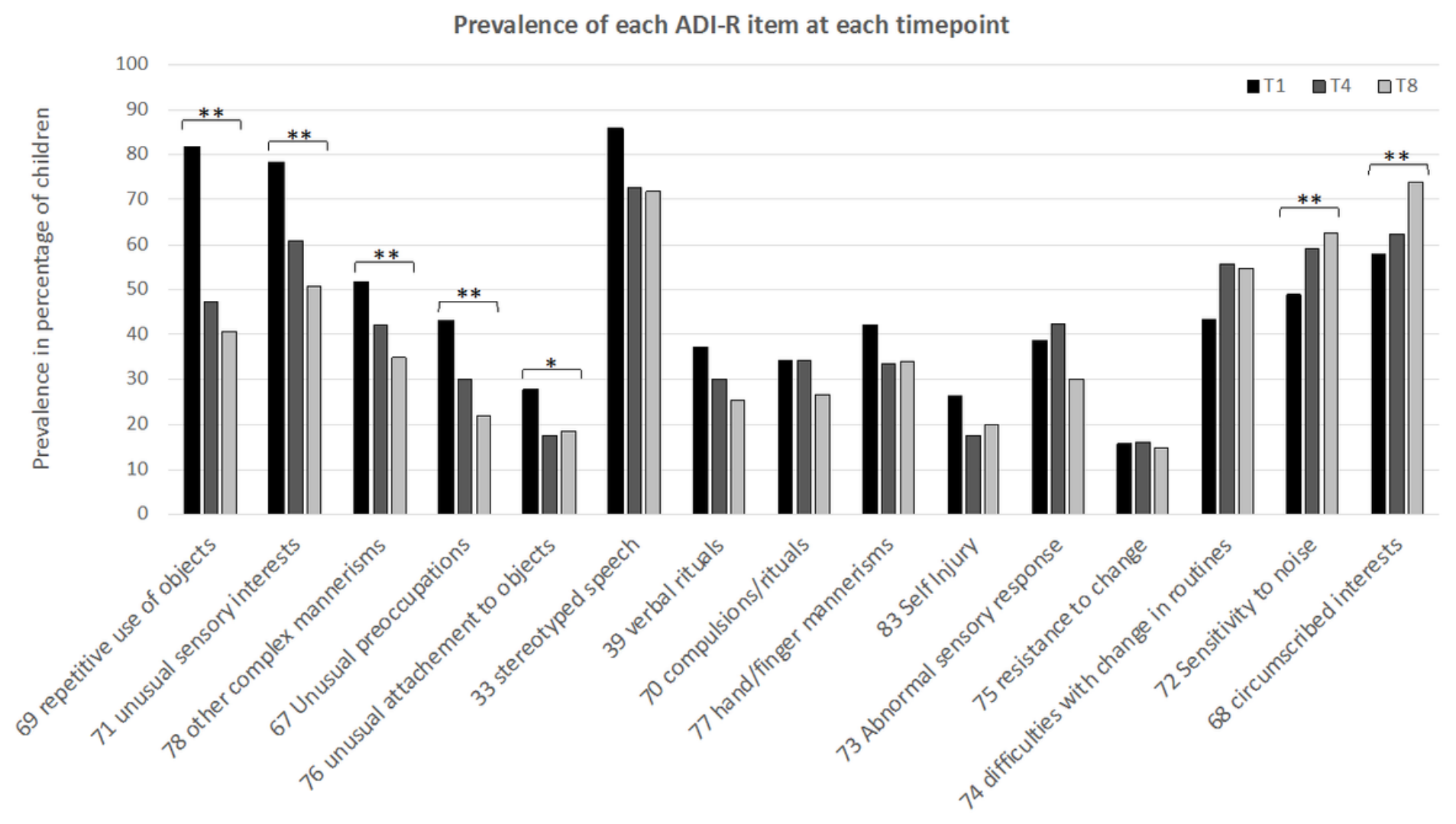

Figure 1

Prevalence of each ADI-R item at each time point. **=item with a significant effect of age after Bonferroni corrections. * item with a significant effect of age after Benjamini-Hochberg corrections. $T 1=$ time of Diagnosis, $T 4=6$ years old, $T 8=11$ years old. 


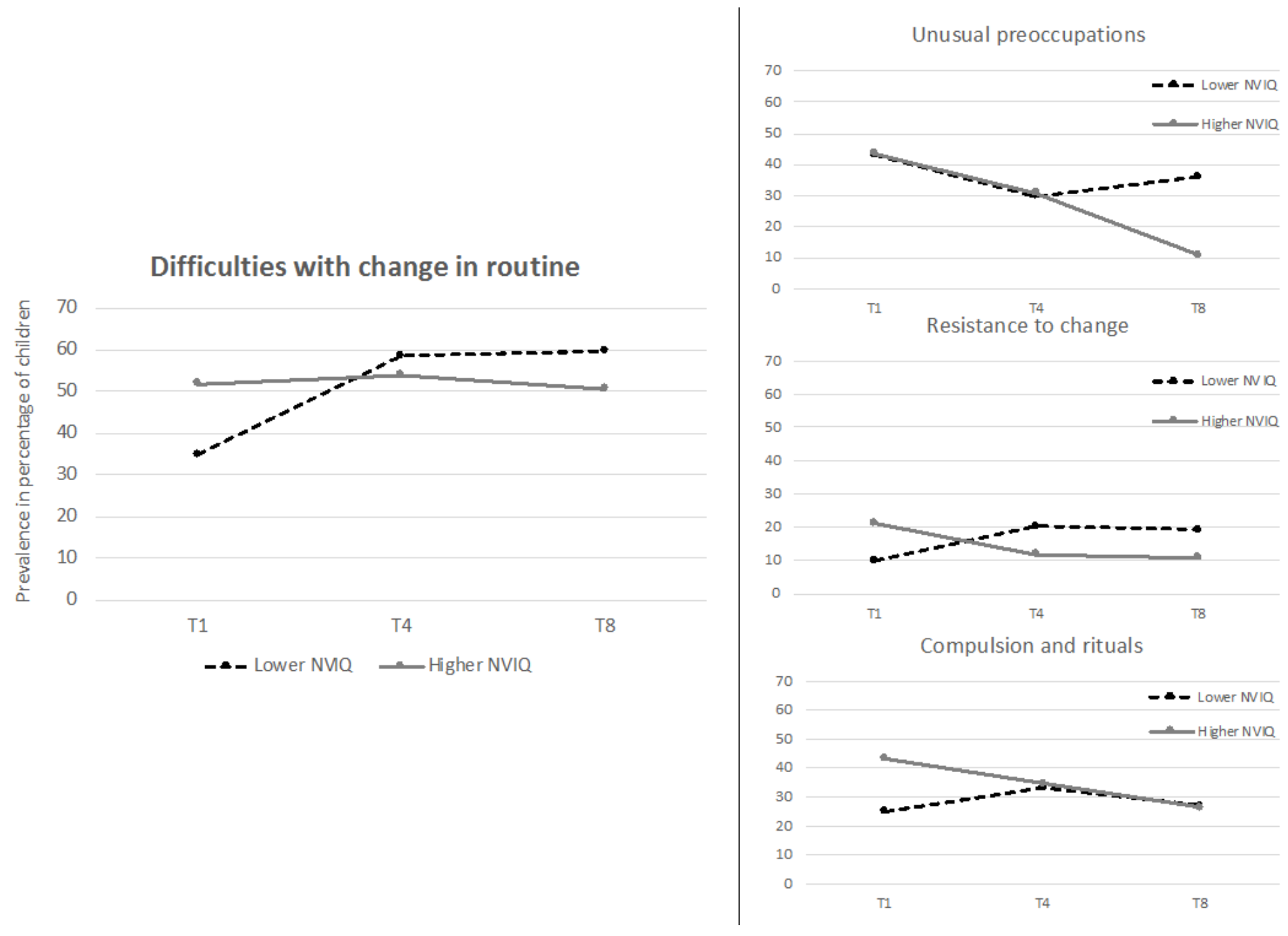

Figure 2

Percentage of children at each time point for each item presenting a significant interaction between age and NVIQ. The item on the left was significant following Bonferroni correction and the three additional items on the right were significant only when using Benjamini-Hochberg corrections. Lower NVIQ $=<93$; $n=101$, Higher NVIQ $=>93 ; n=104 . T 1=$ time of Diagnosis, $T 4=6$ years old, $T 8=11$ years old. 


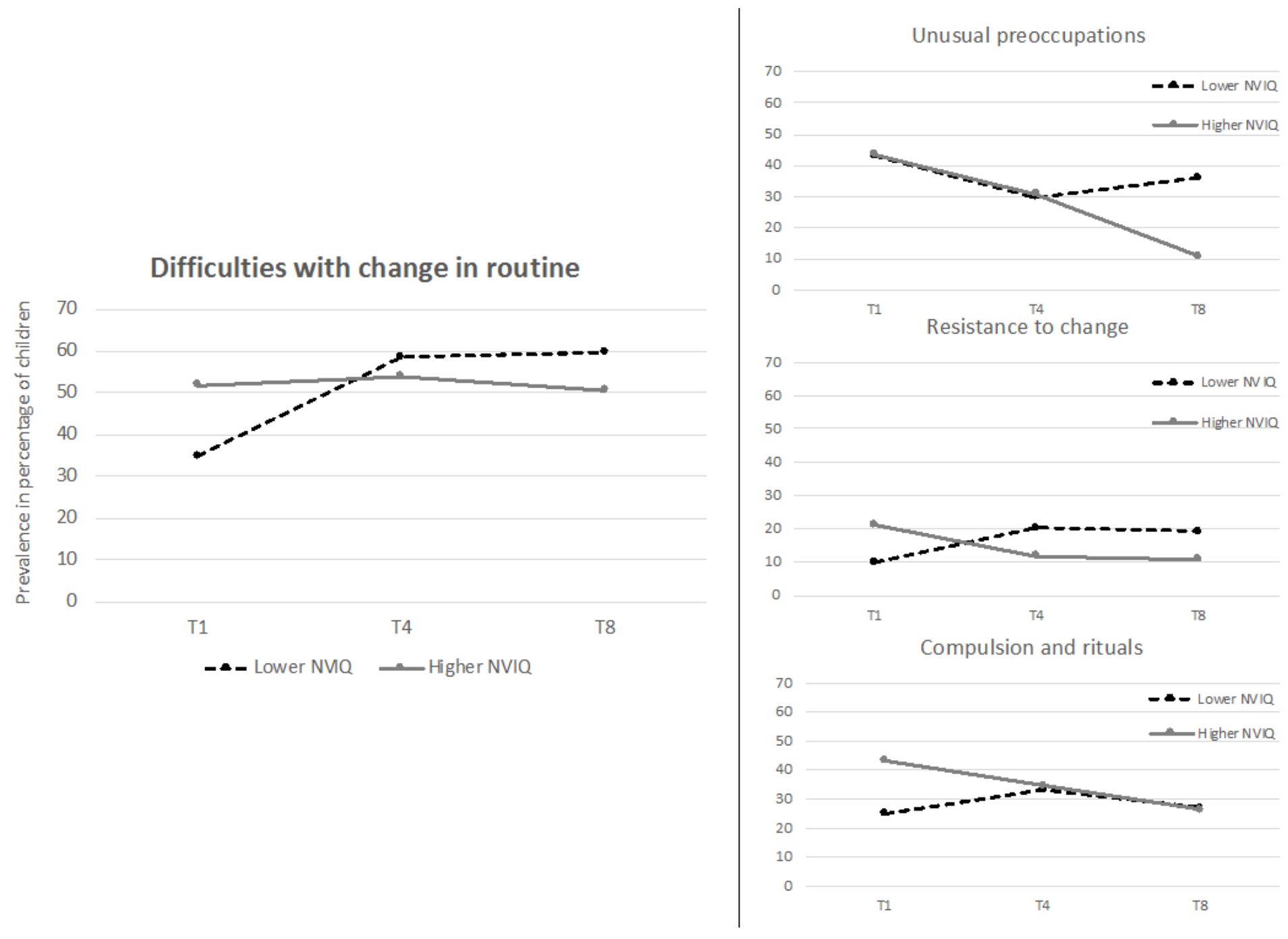

Figure 2

Percentage of children at each time point for each item presenting a significant interaction between age and NVIQ. The item on the left was significant following Bonferroni correction and the three additional items on the right were significant only when using Benjamini-Hochberg corrections. Lower NVIQ $=<93$; $\mathrm{n}=101$, Higher $\mathrm{NVIQ}=>93 ; \mathrm{n}=104$. $\mathrm{T} 1=$ time of Diagnosis, $T 4=6$ years old, $T 8=11$ years old.

\section{Supplementary Files}

This is a list of supplementary files associated with this preprint. Click to download.

- Supplementarymaterial.docx

- Supplementarymaterial.docx 